1995-08-01

\title{
Electromagnetic boundary conditions and differential forms
}

\author{
Karl F. Warnick \\ warnick@byu.edu \\ Richard H. Selfridge \\ selfridge@ee.byu.edu \\ David V. Arnold
}

Follow this and additional works at: https://scholarsarchive.byu.edu/facpub

Part of the Electrical and Computer Engineering Commons

\section{Original Publication Citation}

Warnick, K. F., R. H. Selfridge, and D. V. Arnold. "Electromagnetic Boundary Conditions and Differential Forms." Microwaves, Antennas and Propagation, IEE Proceedings - 142.4 (1995): 326-32

\section{BYU ScholarsArchive Citation}

Warnick, Karl F.; Selfridge, Richard H.; and Arnold, David V., "Electromagnetic boundary conditions and differential forms" (1995). Faculty Publications. 686.

https://scholarsarchive.byu.edu/facpub/686

This Peer-Reviewed Article is brought to you for free and open access by BYU ScholarsArchive. It has been accepted for inclusion in Faculty Publications by an authorized administrator of BYU ScholarsArchive. For more information, please contact ellen_amatangelo@byu.edu. 


\title{
Electromagnetic boundary conditions and differential forms
}

\author{
K.F. Warnick \\ R.H. Selfridge \\ D.V. Arnold
}

Indexing terms: Differential forms, Electromagnetic boundary conditions, Projection aperator, Vector analysis

Abstract: A new representation for electromagnetic boundary conditions involving a boundary projection operator defined using the interior and exterior products of the calculus of differential forms is developed. This operator expresses boundary conditions for fields represented by differential forms of arbitrary degree. With vector analysis, the field intensity boundary conditions require the cross product, whereas the flux boundary conditions use the inner product. With differential forms, the field intensity and flux density boundary conditions are expressed using a single operator. This boundary projection operator is readily applied in practice, so that this work extends the utility of the calculus of differential forms in applied electromagnetics.

\section{Introduction}

In this paper a new formulation is derived for the boundary conditions at a discontinuity in the electromagnetic field using differential forms. The utility of the calculus of differential forms in electromagnetic field (EM) theory has been demonstrated by Deschamps [1], Baldomir [2], Schleifer [3], Thirring [4], Burke [5, 6] and others. The intent of this paper is to extend the range of engineering problems for which differential forms are useful by providing a practical means of working with boundary conditions.

Thirring [4] and Burke $[5,6]$ treat boundary conditions using the calculus of differential forms. Thirring's approach is similar to ours, but we extend his methods by introducing a boundary projection operator to express boundary conditions for forms of arbitrary degree in a space of arbitrary dimension. The expressions for junction conditions on field intensity and flux density, for example, are identical in form.

For many electromagnetic quantities, the vector representation and the representation as a differential form are duals, so that their components differ only by metrical coefficients. This is not the case for the surface current and charge density twisted forms yielded by the boundary projection operator. It is simpler to compute, for example, total current through a path using the surface

(C) IEE, 1995

Paper 2003H (E11, E12), first received 29th November 1994 and in revised form 23rd March 1995

The authors are with Brigham Young University, Department of Electrical and computer Engineering, 459 Clyde Building, Provo UT 84602, USA

326 current twisted 1-form than using the usual surface current vector.

In Section 2 we derive an expression for bondary sources at a field discontinuity using the boundary projection operator. In Section 3 we provide a simple computational example to illustrate the method. Due to the unfamilarity of most engineers with differential forms and associated notation, our treatment is more elementary than is usual. Accordingly, we also provide an introduction to the interior product and twisted forms in an Appendix. This work shows that the calculus of differential forms is useful for practical EM problems involving boundary conditions.

\section{Boundary conditions}

In this section we derive an expression for sources on a boundary where the electromagnetic field is discontinuous. We find that generalised boundary conditions can be given using a boundary projection operator. We then discuss the resulting boundary conditions for magnetic field intensity and electric flux density.

\subsection{Representing surfaces with 1 -forms}

In an $n$-dimensional space, a 1 -form is represented graphically by $(n-1)$-dimensional hypersurfaces. In $n$ space, a boundary is also an $(n-1)$-dimensional hypersurface. Thus, we can use 1 -forms to represent boundaries. If a continuous function $f\left(x_{1}, \ldots, x_{n}\right)$ vanishes (or is constant) along a boundary, then the 1-form df graphically has a surface that lies on the boundary. The surface of a paraboloid reflector antenna, for example, is given by $-x^{2}-y^{2}+a z=0$, so that the unnormalised boundary 1 -form is $-2 x d x-2 y d y+a d z$. A randomly rough surface is written $-h(x, y)+z=0$, giving the boundary 1-form $-d h+d z$.

For the remainder of this paper we will use the notation

$$
n \equiv \frac{d f}{|d f|}=\frac{d f}{\sqrt{ }(d f\lrcorner d f)}
$$

where $\lrcorner$ is the interior product, which is discussed in the Appendix. The 1 -form $n$ is dual to the usual surface normal vector $\hat{n}$.

This material is based in part upon work supported under a National Science Foundation Graduate Fellowship to KFW.

IEE Proc.-Microw. Antennas Propag., Vol. 142, No. 4, August 1995 
2.2 Derivation of the boundary projection operator In three dimensions, let $E$ be the electric field intensity 1 -form, $H$ the magnetic field intensity 1 -form, $D$ the electric flux density 2 -form, $B$ the magnetic flux density 2 -form, $J$ the electric current density 2-form and $\rho$ the charge density 3-form. Then Maxwell's laws are

$$
\begin{aligned}
d E & =-\frac{\partial}{\partial t} B \\
d H & =\frac{\partial}{\partial t} D+J \\
d D & =\rho \\
d B & =0
\end{aligned}
$$

Each equation equates the exterior derivative of a differential form to the sum of a source and a nonsingular field term, of which one or both may vanish. Recognising that Maxwell's laws have a common form, we can derive an expression for the boundary sources that is the same for both field intensity (1-forms) and flux density (2-forms).

Let $\alpha$ be a $p$-form with $p<n$ (where $n$ is the dimension of space) that represents a field with a $(p+1)$-form $\beta$ as a source, so that

$$
d \alpha=\gamma+\beta
$$

where $\gamma$ is nonsingular. Let $f=0$ represent a boundary, where $f$ is $C^{1}$ and vanishes only along the boundary. Let $\alpha$ equal $\alpha_{2}$ for $f>0$ and $\alpha_{1}$ for $f<0$.

We can write $\alpha=\left(\alpha_{2}-\alpha_{1}\right) \theta(f)+\alpha_{1}$, where $\theta$ is the unit step function. Then

$$
\begin{aligned}
\gamma+\beta & =d\left\{\left(\alpha_{2}-\alpha_{1}\right) \theta(f)+\alpha_{1}\right\} \\
& =\delta(f) d f \wedge\left(\alpha_{2}-\alpha_{1}\right)+\theta(f) d\left(\alpha_{2}-\alpha_{1}\right)+d \alpha_{1} \\
& =\tilde{\delta}(f) n \wedge\left(\alpha_{2}-\alpha_{1}\right)+\theta(f) d\left(\alpha_{2}-\alpha_{1}\right)+d \alpha_{1}
\end{aligned}
$$

where $\delta$ is the Dirac delta function and $\delta(f)$ is $\delta\left(x^{1}-x_{0}^{\mathbf{t}}\right)$ $\cdots \delta\left(x^{n}-x_{0}^{n}\right)$ such that the point $\left(x_{0}^{1}, \ldots, x_{0}^{n}\right)$ lies on the boundary and $\delta(f)=\tilde{\delta}(f) / \sqrt{ }(d f\lrcorner d f)$. The singular parts of both sides of eqn. 4 must be equal, so that

$$
\beta^{\prime}=\tilde{\delta}(f) n \wedge\left(\alpha_{2}-\alpha_{1}\right)
$$

where $\beta^{\prime}$ is the singular part of $\beta$, representing the boundary source along $f=0$. Since the source $\beta^{\prime}$ is confined to the boundary, it can be written [4]

$$
\beta^{\prime}=\tilde{\delta}(f) n \wedge \beta_{s}
$$

where $\beta_{s}$ is a $p$-form, the restriction of $\beta^{\prime}$ to the boundary. Integrating eqns. 5 and 6 , over a region containing the boundary shows that the equality

$$
n \wedge \beta_{s}=n \wedge\left(\alpha_{2}-\alpha_{1}\right)
$$

must hold on the boundary. We then take the interior product of both sides of eqn. 7 by $n$ and apply the identity 30 to obtain

$$
\begin{aligned}
n\lrcorner\left(n \wedge\left(\alpha_{2}-\alpha_{1}\right)\right) & =n\lrcorner\left(n \wedge \beta_{s}\right) \\
& \left.=(n\lrcorner n) \wedge \beta_{s}-n \wedge n\right\lrcorner \beta_{s}
\end{aligned}
$$

Because $n$ is normalised, $n\lrcorner n=1$. Since $\beta_{s}$ is by definition confined to the boundary, $n\lrcorner \beta_{s}=0$. Graphically, the surfaces of $\beta_{s}$ are perpendicular to the boundary because $\beta_{s}$ can be contain no factor proportional to $n$. Applying $n\lrcorner \beta_{s}=0$ to eqn. 8, we have

$$
\left.\beta_{s}=n\right\lrcorner\left(n \wedge\left(\alpha_{2}-\alpha_{1}\right)\right)
$$

which is the central result of this paper.

IEE Proc-Microw. Antennas Propag., Vol. 142, No. 4, August 1995
Eqn. 9 applies to both nontwisted and twisted forms. As noted below, electromagnetic sources are conveniently represented by twisted forms. If $\beta_{s}$ is a twisted form, we must provide an outer orientation (see Appendix and Burke [6]) for $\beta_{s}$. The orientation is given by

$$
\left\{\left(\beta_{s}, \Omega_{s}\right)\right\} \wedge n \wedge \beta_{s}=\Omega
$$

where $\left\{\left(\beta_{s}, \Omega_{s}\right)\right\}$ is a nontwisted $(n-p-1)$-form specifying the outer orientation of $\beta_{s}$ and $\Omega$ is a volume element ( $n$-form) serving as an orientation for the surrounding $n$-space. $\Omega_{s}$ is a volume element in the boundary, but need not be found in order to obtain the outer orientation of $\beta_{s}$. Given any boundary, there are two possible choices for $n$. The orientation specified by eqn. 10 is easily seen to be independent of that choice. In right-handed coordinates, an equivalence can be made between inner and outer orientations, and the orientation of $\beta_{s}$ can be taken as that of the $(p+1)$-form $n \wedge \beta_{s}$.

In Sections 2.4 and 2.5 , where eqn. 9 is specialised to surface current and surface charge densities, we provide a simpler means for orienting surface sources. We find that the need for an orientation for $\beta_{\text {s }}$ corresponds precisely to conventions used with the vector calculus when integrating $J_{s}$ and the scalar surface charge density $q_{s}$.

Since eqn. 9 applies to any situation for which a law of the form 3 is valid, we can use Maxwell's laws 2 to write at a boundary $f=0$,

$$
\begin{aligned}
& n\lrcorner\left(n \wedge\left(E_{2}-E_{1}\right)\right)=0 \\
& n\lrcorner\left(n \wedge\left(H_{2}-H_{1}\right)\right)=J_{s} \\
& n\lrcorner\left(n \wedge\left(D_{2}-D_{1}\right)\right)=\rho_{s} \\
& n\lrcorner\left(n \wedge\left(B_{2}-B_{1}\right)\right)=0
\end{aligned}
$$

where $J_{s}$ is the surface current twisted 1 -form and $\rho_{\mathrm{s}}$ is the surface charge twisted 2-form. In four-space we have $d F=0$ and $d \star F=j$, where $F=B+E \wedge d t, \star F=D$ $-H \wedge d t$ and $j=\rho-J \wedge d t$. We can express all four boundary conditions as

$$
\begin{aligned}
& n\lrcorner\left(n \wedge\left(F_{2}-F_{1}\right)\right)=0 \\
& n\lrcorner\left(n \wedge\left(\star F_{2}-\star F_{1}\right)\right)=j_{s}
\end{aligned}
$$

where $j_{s}=\rho_{s}-J_{s} \wedge d t$ and units are suitably normalised.

The operator $n\lrcorner n \wedge$ might be termed the boundary projection operator. Graphically, this operator removes the component of a form with surfaces parallel to the boundary. The boundary projection of a 1-form has surfaces perpendicular to the boundary. The boundary projection of a 2-form has tubes perpendicular to the surface at every point.

\subsection{Decomposition of forms at a boundary}

Any form $\omega$ of degree less than the dimension of the space we are working in can be decomposed using the boundary projection operator and its complementary operation $\left.\star^{-1} n\right\lrcorner n \wedge \star$, so that

$$
\left.\omega=n\lrcorner(n \wedge \omega)+\star^{-1}[n\lrcorner(n \wedge \star \omega)\right]
$$

where the second term on the right-hand side is the component of $\omega$ with surfaces parallel to the boundary. This identity can be proved by expressing $\omega$ in terms of orthonormal 1-forms $d x^{i}$ at a point, verifying eqn. 13 for $n=d x^{i}$ and extending to the general case $n=n_{i} d x^{i}$, where $\sum_{i}\left(n_{i}\right)^{2}=1$ using the linearity of the exterior and interior products. The operator $\left.\star^{-1} n\right\lrcorner n \wedge \star$ can be used to obtain the arbitrary part of a field at a boundary.

We discuss special cases of the boundary projection operator for 1-forms and 2-forms below. 
2.4 Surface current

Surface current is represented by a twisted 1-form. Fig. 1 shows how this 1-form is obtained from the magnetic
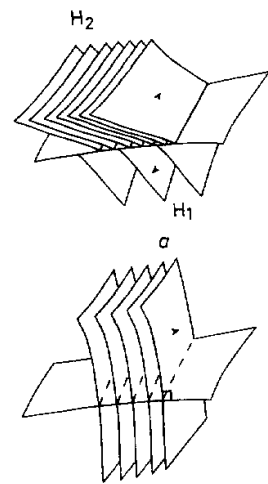

$c$
$\mathrm{H}_{2}-\mathrm{H}_{1}$
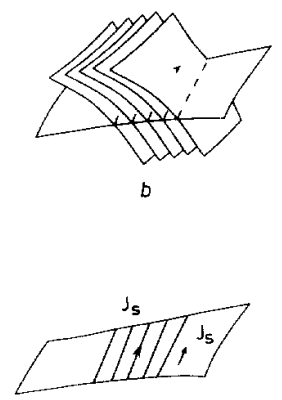

$d$
Fig. 1 (a) A boundary with discontinuous magnetic field. (b) The field $\left(H_{2}-H_{1}\right)$, (c) The 1-form $\left.n\right\lrcorner\left(n \wedge\left(H_{2}-H_{1}\right)\right)$. (d) The restriction of this l-form to the boundary, along with the corresponding vector $J_{s}$

field intensity at a boundary (Fig. $1 a$ ). Fig. $1 b$ shows the 1 -form $\left(H_{2}-H_{1}\right)$. (Note that the 1 -forms $H_{2}$ and $H_{1}$ are both defined above and below the boundary even though each represents the field on one side of the boundary only. Thus, we can consider $H_{2}-H_{1}$ as being defined for all space, even though only its value on the boundary is of interest.) Fig. $1 c$ shows $n\lrcorner\left(n \wedge\left(H_{2}-H_{1}\right)\right)$. Fig. $1 d$ is the restriction of this 1-form to the boundary, along with the corresponding vector $J_{s}$.

Graphically, the boundary projection operator removes any component of $\mathrm{H}_{2}-\mathrm{H}_{1}$ with surfaces parallel to the boundary. Physically, this expresses both the requirement that surface current can only flow along the boundary and the arbitrariness of the normal component of $H_{2}-H_{1}$ at the boundary.

Most forms in EM theory, such as $E, H, D$ and $B$, are dual to the corresponding vector quantity, so that components differ only by metrical coefficients. The twisted surface current 1-form $J_{s}$, however, is not the dual of the vector $J_{s}$. The nontwisted 1 -form with the same components as $J_{s}$ does not satisfy the simple definition given below in definition 14

The surface current 1-form $J_{s}$ can be defined in terms of the, flow vector $v$ of a surface charge distribution $\rho_{s}$. The current density 2 -form $J$ is $J=v\lrcorner \rho$. The surface current density is $\left.J_{s}=-v\right\lrcorner \rho_{\mathrm{s}}$. If $\rho_{s}=q d x d y$ and the flow field $v=v \hat{y}$, then $\left.J_{s}=-v \hat{y}\right\lrcorner q d x d y=q v d x$. The 1-form dual to $J_{s}$ would be $q v d y$.

The integral of the surface current density over a path should yield the total current through the path. The 1 -form $J_{s}$ as obtained using the boundary projection operator satisfies this definition

$$
I=\int_{P} J_{s}
$$

where $P$ is a path. The sense of $I$ is with respect to the direction of the 2-form $n \wedge s$, where $s$ is the 1-form dual to the tangent vector $s$ of $P$ (so that $s$ is a 1-form with surfaces perpendicular to the path $P$ and oriented in the direction of integration).

The simple integral in eqn. 14 replaces a much more cumbersome vector expression. The total current through
$P$ using the usual surface current vector is

$$
I=\int_{P} J_{s} \cdot(\hat{n} \times d s)
$$

where the sense of $I$ is relative to the direction of $\hat{n} \times d s$. Note that this is the same as the reference direction $n \wedge s$ of eqn. 14.

The integral in eqn. 14 is evaluated in practice by the method of pullback. If a surface $S$ is parameterised by $x=\alpha_{1}(u, v), y=\alpha_{2}(u, v), z=\alpha_{3}(u, v)$ where $u$ and $v$ range over some subset $T$ of the $u-v$ plane, then the integral of

$$
\omega=\omega_{1}(x, y, z) d x+\omega_{2}(x, y, z) d y+\omega_{3}(x, y, z) d z
$$

over $S$ is

$$
\begin{aligned}
\int_{S} \omega= & \int_{T} \alpha^{*} \omega \\
= & \int_{T} \omega_{1}\left(\alpha_{1}, \alpha_{2}, \alpha_{3}\right) d \alpha_{1}+\omega_{2}\left(\alpha_{1}, \alpha_{2}, \alpha_{3}\right) d \alpha_{2} \\
& +\omega_{3}\left(\alpha_{1}, \alpha_{2}, \alpha_{3}\right) d \alpha_{3}
\end{aligned}
$$

where the superscript ${ }^{*}$ denotes the pullback operation. After pullback, the integrand becomes a 1-form in $d u$ and $d v$. Partial derivatives of the coordinate transformation enter naturally via the exterior derivatives of $\alpha_{1}, \alpha_{2}$ and $\alpha_{3}$. Eqn. 16 is easily generalised to integrals of 2 -forms.

\subsection{Surface charge}

Surface charge density due to discontinuous electric flux density at a boundary is represented by the twisted 2 -form $\left.\rho_{s}=n\right\lrcorner\left(n \wedge\left(D_{2}-D_{1}\right)\right)$. The boundary projection operator removes any component of $D_{2}-D_{1}$ with tubes parallel to the boundary, as shown in Fig. 2.

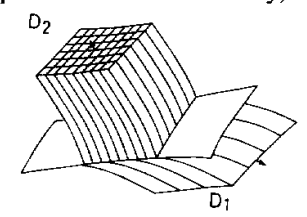

$a$

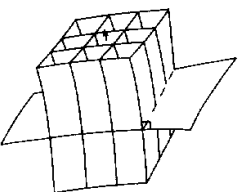

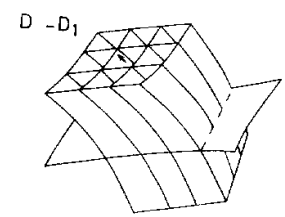

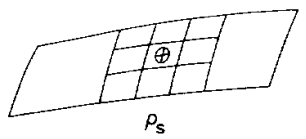

Fig. 2 (a) A boundary with discontinuous electric fux. (b) The field $\left(D_{2}-D_{1}\right)$. (c) The 2-form $\left.n\right\lrcorner\left(n \wedge\left(D_{2}-D_{1}\right)\right)$. (d) The restriction of this 2 -form to the boundary

The 2-form $\rho_{s}$ obtained using the boundary projection operator differs from the usual value $q_{s}=\hat{n} \cdot\left(\boldsymbol{D}_{2}-D_{1}\right)$ because $\rho_{s}$ is a 2 -form, whereas $q_{s}$ is a scalar. The total charge on an area $A$ of a boundary with surface charge $\rho_{s}$ is

$$
Q=\int_{A} \rho_{s}
$$

$Q$ is positive for positive surface charge if the 2-form $\omega$ that satisfies $n \wedge \omega=\Omega$ also satisfies $\int_{A} \omega>0$, where $\Omega$ is the standard volume element, $d x, d y d z$ in rectangular coordinates. This corresponds exactly to the convention

IEE Proc.-Microw. Antennas Propag., Vol. 142, No. 4, August 1995 
of choosing $d S$ in $Q=\int_{\mathrm{A}} q_{\mathrm{s}} d S$ (where $q_{\mathrm{s}}$ is the usual surface charge density scalar) such that $\int_{A} d S$ is positive. The sign of the charge represented by $\rho_{s}$ can also be found by computing $n \wedge \rho_{s} / \Omega$.

\subsection{Comparison to Burke's pullback method}

Burke [6] derives an expression for boundary sources using the method of pullback. In his notation, boundary conditions have the form $[H]=J_{s}$ and $[D]=\rho_{s}$. The square brackets denote the sum of the pullback of $H$ above the boundary to the boundary and the pullback of $H$ below the boundary to the boundary, so that

$$
J_{s}=[H] \equiv \alpha_{2}^{*} H_{2}+\alpha_{1}^{*} H_{1}
$$

where $\alpha_{2}$ and $\alpha_{1}$ are functions of the space above and below the boundary into the boundary.

The pullback method has a concise and elegant proof in [6]. By transforming to a coordinate system $x^{1}, \ldots, x^{n}$ such that a boundary is given by $x^{1}=0$, it can be shown that Burke's formulation is equivalent to $n\lrcorner n \wedge$ for $n=d x^{1}$. Although the pullback boundary conditions are mathematically very natural, the boundary projection operator has the advantage that (as with the usual vector formulation) the boundary sources are always expressed in the same coordinates as the fields.

\section{Example}

A conducting boundary lies along the surface $z=\cos y$. Above the boundary the magnetic field is $\mathrm{H}_{2}=\mathrm{Hdx}$. Below the field is zero. This is shown in Fig. 3a.

We can represent the surface by $f(x, y, z)$ $=z-\cos y=0$. Computing the normalised exterior derivative of this function,

$$
\begin{aligned}
n & =\frac{d(-\cos y+z)}{|d(-\cos y+z)|} \\
& =\frac{\sin y d y+d z}{\sqrt{ }\left(1+\sin ^{2} y\right)}
\end{aligned}
$$

The boundary projection of $\mathrm{H}_{2}$ is

$$
\begin{aligned}
J_{s} & =n\lrcorner n \wedge H_{2} \\
& \left.=\frac{H}{1+\sin ^{2} y}(\sin y d y+d z)\right\lrcorner(\sin y d y d x+d z d x) \\
& =\frac{H}{1+\sin ^{2} y}\left(d x+\sin ^{2} y d x\right) \\
& =H d x
\end{aligned}
$$

Fig. $3 c$ shows the 1-form $J_{s}=H d x$ restricted to the boundary. The direction of the arrow along the lines of $H d x$ is the orientation of the 2 -form $n \wedge J_{s}$

Compare the final expression for $J_{s}$ in eqn. 20 to the vector surface current obtained for the same field and boundary, $J_{s}=H / \sqrt{ }\left(1+\sin ^{2} y\right)(\hat{y}-\sin y \hat{z})$. The differential form $J_{s}=H d x$ indicates clearly that the total current crossing a path in the boundary is simply the extent of the path in the $x$ direction scaled by the factor $H$. This is not obvious from the vector expression.

\section{Conclusions}

The boundary projection operator allows one to express electromagnetic boundary conditions using differential forms, using the same operator for both field intensity and flux density. The different appearances of the field
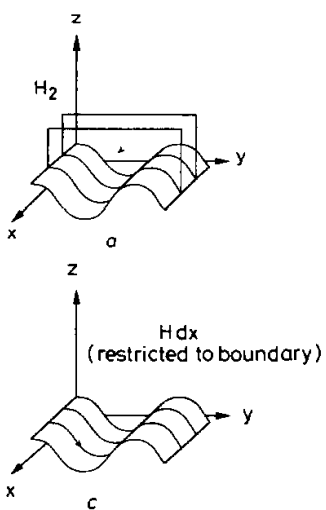

Fig. 3 (a) The boundary $-\cos y+z=0$ with magnetic field $\mathrm{H}_{2}=$ $H d x$ above the boundary. The field is zero below the boundary. (b) The 1-form $H d x$ in 3-space. (c) The 1-form $H d x$ restricted to the boundary

intensity and flux density boundary conditions expressed using vector analysis are merely an artifact of the mathematical language. The differential forms for boundary sources obtained via the boundary projection operator differ from the vectors obtained by standard methods. The surface current 1 -form $J_{s}$, for example, has a more natural definition than the usual surface current vector $J_{s}$. This $1-$ form is readily integrated to yield total current over a path, whereas a vector perpendicular to the path and tangent to the boundary is required to evaluate the integral for total current using the surface current vector. The surface current vector also obscures intuitive properties of the boundary source that are clearly evident when the source is represented by the 1 -form $J_{s}$. Graphically, a source form is the intersection of the field forms with the boundary, for both the field intensity and flux density cases.

This method for representing boundary conditions can be easily applied in practical problems, and so helps to open the way for the use of differential forms on a regular basis in engineering electromagnetics.

\section{References}

1 DESCHAMPS, G.A.: 'Electromagnetics and differential forms', IEEE Proc., 1981, 69, (6), pp. 676-696

2 BALDOMIR, D.: 'Differential forms and electromagnetism in 3dimensional Euclidean space $\mathbf{R}^{3}$, IEE Proc., 1986, 133, (3), pp. 139 143

3 SCHLEIFER, N.: 'Differential forms as a basis for vector analysis with applications to electrodynamics', Amer. J. Phys., 1983, 51, (12) pp. $1139-1145$

4 THIRRING, W.: 'Classical field theory' (Springer-Verlag, New York, 1978)

5 BURKE, W.L.: 'Manifestly parity invariant electromagnetic theory and twisted tensors', J. Math. Phys., 1983, 24, (1), pp. 65-69

6 BURKE, W.L.: 'Applied differential geometry' (Cambridge University Press, Cambridge, 1985), pp. 79-80, 183-200, 192-193, 279282

7 BAMBERG, P., and STERNBERG, S.: 'A course in mathematics for students of physics'. Vol. II (Cambridge University Press, Cambridge, 1988), pp. 621-626

8 MISNER, C., THORNE, K., and WHEELER, J.A.: 'Gravitation'

(Freeman, San Francisco, 1973), pp. 90-129

\section{Appendix. The interior product; twisted forms}

In this Appendix we provide an introduction to the interior product and twisted forms; $[6,7]$ and others provide more comprehensive treatments of the same 
topics. The interior product combines a vector (or by abuse of notation, a 1-form) and a $p$-form to produce a $(p-1)$-form. Twisted forms differ from non-twisted forms in that a twisted has an outer orientation rather than an inner orientation and so changes sign relative to a nontwisted form under reflection.

\subsection{The interior product}

A differential such as $d x$ is traditionally viewed as an infinitesimal increment of the coordinate $x$. From the differential geometric point of view, $d x$ is actually a basis element of $R^{n *}$, the space of linear functions from vectors in $R^{n}$ into $R$. A basis for $R^{n *}$ is $d x^{1}, \ldots, d x^{n}$, which act on basis vectors $d x_{1}, \ldots, d x_{n}$ of $R^{n}$ (written $\partial / \partial x^{1}, \ldots, \partial / \partial x^{n}$ by the mathematician) as

$$
d x^{i}\left[d x_{j}\right]= \begin{cases}1 & i=j \\ 0 & i \neq j\end{cases}
$$

where the square brackets indicate that the differential is a function with the vector inside the brackets as its argument. By linearity, eqn. 21 extends to arbitrary 1-forms and vectors.

This definition of 1 -forms leads directly to the graphical representation of Misner et al. [8]. 1-forms become surfaces in space. The value of a 1-form operated on a vector is the number of surfaces of the 1-form pierced by the vector (see Reference 8 for extensive figures illustrating this point).

Integration can be defined naturally by using the 1-form to be integrated as a linear operators on vectors that specify the region of integration. If $P$ is an arbitrary path in space broken polygonally into $n$ small vectors $v_{i}$, then the integral of the 1 -form $\omega$ over $P$ is

$$
\int_{P} \omega=\lim _{n \rightarrow \infty} \sum_{i=1}^{n} \omega\left[\boldsymbol{v}_{i}\right]
$$

where the vectors $v_{i}$ become infinitesimal in the limit. Eqn. 22 shows that the graphical representation of forms as operators on vectors extends to integration of forms over paths: the integral of a 1-form over a path is the number of surfaces of the 1 -form pierced by the path.

The interior product is written $v\lrcorner \omega$ where $v$ is a vector and $\omega$ is a form. If $\omega$ is a 1 -form, the interior product of $v$ and $\omega$ is

$$
\boldsymbol{v}\lrcorner \omega \equiv \omega[\boldsymbol{v}]
$$

which is simply the definition of $\omega$ as a linear operator acting on the vector $v$.

The interior product of a vector and a 2-form follows from the definition of a 2 -form as the antisymmetrised tensor product of two 1-forms, so that $a \wedge b=$ $a \otimes b-b \otimes a$, where $a$ and $b$ are 1-forms and $\otimes$ is the tensor product. The tensor product of 1 -forms $a$ and $b$ is defined so that if $v$ and $w$ are vectors, $a \otimes b$ is a function of two vectors, and $a \otimes b[v, w]=a[v] b[w]$, which is a real number. If we operate $a \wedge b$ on one vector $v$ rather than two, we obtain the 1-form $a[v] b-b[v] a$. This is the interior product $v\lrcorner(a \wedge b)$. The interior product of $\hat{x}$ and $d x \wedge d y$ is

$$
\begin{aligned}
\hat{x}\lrcorner(d x \wedge d y) & =d x \wedge d y[\hat{x}, \cdot] \\
& =d x[\hat{x}] \otimes d y-d y[\hat{x}] \otimes d x \\
& =d y
\end{aligned}
$$

The interior product of a vector $v$ and a $p$-form $a d x^{i_{1}} \wedge$ $\cdots \wedge d x^{i p}$ can be obtained for arbitrary $p$ by repeated application of the definition of the exterior product as the antisymmetrised tensor product. When $a d x^{i_{1}} \wedge \cdots \wedge d x^{i_{p}}$ is expanded in terms of the tensor product, the first factor of each term operates on the vector $\boldsymbol{v}$. This can be written conveniently using the determinant

$$
v\lrcorner \omega=a \operatorname{det}\left[\begin{array}{ccc}
d x^{i_{1}}[v] & \cdots & d x^{i_{p}}[v] \\
d x^{i_{1}} & \cdots & d x^{i_{p}} \\
\vdots & & \vdots \\
d x^{i_{1}} & \cdots & d x^{i_{p}}
\end{array}\right]
$$

where the top row of 1 -forms operate on $v$ and the determinant then evaluates to a $(p-1)$-form.

We can now define the interior product $\alpha\lrcorner \omega$ of a 1 -form $\alpha$ and a $p$-form $\omega$. The metric $g_{i j}$ must be used to convert a 1 -form to its dual vector ('raising the index'). With the Euclidean metric, a 1-form and its dual have the same components, so that we can write in $R^{3}$,

$$
(a d x+b d y+c d z)\lrcorner \omega=(a \hat{x}+b \hat{y}+c \hat{z})\lrcorner \omega
$$

In curved spaces or curvilinear coordinates, the definition becomes

$$
\left.\left.\alpha_{i} d x^{i}\right\lrcorner \omega=g^{i j} \alpha_{j} d x_{i}\right\lrcorner \omega
$$

where $g^{i j}$ is the inverse of the metric $g_{i j}$ and repeated indices are summed over $1, \ldots, n$ with $n$ the dimension of space, $g^{i j} \alpha_{j} d x_{i}$ is the vector dual to the 1 -form $\alpha$. The components of the metric for spherical coordinates are

$$
g_{i j}=\left[\begin{array}{lll}
1 & & \\
& r^{2} & \\
& & r^{2} \sin ^{2} \theta
\end{array}\right]
$$

which along with eqn. 27 allow the boundary conditions given in Section 2 to be used for geometries with spherical symmetry.

We note in passing that one can also define the interior product of a $p$-form and a $q$-form for arbitrary $p$ and $q$ using relation 4

$$
v\lrcorner \star w=\star(w \wedge v)
$$

where $\star$ is the Hodge star operator. If $\star \star=1$, as is the case for three-dimensional space with a positive definite metric, this can be used to rewrite the boundary projection operator in terms of the exterior product and star operator as $\star\left[\star\left(n \wedge\left(\alpha_{2}-\alpha_{1}\right)\right) \wedge n\right]$.

In view of eqn. 27, the interior product of two 1-forms is simply their inner product. The magnitude $|\alpha|$ of a 1 -form $\alpha$ is then $\sqrt{ }(\alpha\lrcorner \alpha)$ or $\left.\sqrt{ }(\alpha\lrcorner \alpha^{*}\right)$ where the superscript $^{*}$ denotes complex conjugation if the coefficients of $\alpha$ are complex.

The interior product distributes over the exterior product by.

$$
\left.\alpha\lrcorner(\beta \wedge \gamma)=(\alpha\lrcorner \beta) \wedge \gamma+(-1)^{p} \beta \wedge \alpha\right\lrcorner \gamma
$$

where $p$ is the degree of $\beta$.

\subsection{The interior product in $R^{3}$}

For $R^{3}$ with the Euclidean metric the interior product has a simple computational rule. The interior product of a differential $d x$ and a term of an arbitrary form containing $d x$ as a factor is found by moving $d x$ to the left of the 
term, switching the sign of the term each time two differ'entials are interchanged, and then removing the differential $d x$ from the term. If $d x$ is not present in the term, the interior product is zero. For example

$$
\begin{aligned}
3 d x & \lrcorner(d z \wedge d x+2 d y \wedge d z) \\
& =3 d x\lrcorner d z \wedge d x+6 d x\lrcorner d y \wedge d z \\
& =-3 d x\lrcorner d x \wedge d z+0 \\
& =-3 d z
\end{aligned}
$$

This rule can be used to obtain the interior product of a 1 -form and an arbitrary $p$-form.

As noted above, the interior product of two 1-forms is their inner product,

$$
\begin{aligned}
& \left.\left(a_{1} d x+a_{2} d y+a_{3} d z\right)\right\lrcorner\left(b_{1} d x+b_{2} d y+b_{3} d z\right) \\
& \quad=a_{1} b_{1}+a_{2} b_{2}+a_{3} b_{3}
\end{aligned}
$$

The interior product of a 1 -form and a 2 -form is

$$
\begin{aligned}
\left(a_{1} d x\right. & \left.+a_{2} d y+a_{3} d z\right) \\
& -\left(b_{1} d y d z+b_{2} d z d x+b_{3} d x d y\right) \\
= & \left(a_{3} b_{2}-a_{2} b_{3}\right) d x+\left(a_{1} b_{3}-a_{3} b_{1} d y\right. \\
& +\left(a_{2} b_{1}-a_{1} b_{2}\right) d z
\end{aligned}
$$

which compares to the cross-product of vectors with the same components. The exterior product of two 1-forms, the interior product of a 1-form and a 2 -form and the vector cross-product result in the same coefficients. The exterior product of a 1-form and a 2-form, the interior product of two 1-forms and the vector inner product result in the same coefficient. With this identification of operations on forms with vector operations, the rule 30 for $R^{3}$ contains several vector identities as special cases.

\subsection{Examples of the use of the interior product}

The vector Lorentz force law is

$$
\boldsymbol{F}=q(\boldsymbol{E}+\boldsymbol{v} \times \boldsymbol{B})
$$

where $v$ is the velocity of the charge $q$. Written using differential forms, this law becomes

$$
F=q(E-v\lrcorner B)
$$

where $F$ is the force field 1-form. The vector current density is given by

$$
J=v q
$$

where $v$ is the velocity field of a volume charge distribution with scalar density $q$. Writing the same charge distribution as the 3 -form $\rho$, this becomes

$$
J=\boldsymbol{v}\lrcorner \rho
$$

If $\rho=q d x d y d z$ and the flow field $v=v \hat{x}$, for example, then $J=v \hat{x}\lrcorner q d x d y d z=v q d y d z$.

\subsection{Twisted forms}

This Appendix is intended only to provide background in the mathematics of twisted forms for the interested reader. In practice, we agree to use only right-handed coordinates, and the distinction between twisted and nontwisted forms disappears. One can switch between outer and inner orientations at will, using whichever is more appropriate to the quantity at hand. The use of twisted forms in EM theory therefore does not complicate calculations; rather, twisted forms become a graphical tool, adding significantly to the intuition obtainable from pictures of boundary conditions.
A twisted form changes sign under a reflection of the coordinate system relative to a nontwisted form with the same components. Other terms for twisted tensors are oriented, Weyl or odd. Axial vectors or pseudovectors are dual to twisted 1-forms and nontwisted 2-forms in $R^{3}$; polar vectors are dual to nontwisted 1 -forms and twisted 2-forms.

Graphically, each differential form is represented by surfaces in space. There are two possible orientations for each set of surfaces, and so an orientation must be specified in addition to the surfaces. A nontwisted form has an inner orientation, so that each of its surfaces has a perpendicular direction associated with it. The nontwisted 1 -form $d x$ has inner orientation in the $+x$ direction; the 2 -form $d y d z$ obtains a screw sense from the inner orientations of $d y$ and $d z$.

A twisted $p$-form is given an outer orientation rather than an inner orientation. Graphically, an outer orientation for a form $\alpha$ is the orientation of a form consisting of surfaces orthogonal to the surfaces of $\alpha$. In Burke's [6] notation, a twisted form is written as a pair $(\alpha, \Omega)$ of a nontwisted form $\alpha$ and volume element ( $n$-form) $\Omega$. If the twisted form $\alpha$ has degree $p$, its outer orientation $\{(\alpha, \Omega)\}$ is an $(n-p)$-form and is given by

$$
\{(\alpha, \Omega)\} \wedge \alpha=\Omega
$$

Under coordinate reflection, the volume element changes sign, so that outer orientations also must change sign relative to inner orientations. Note that the sign change is relative; under reflection, the orientation of a nontwisted form may reverse while the corresponding twisted form retains its original orientation. This is the case for 2 -forms in $R^{3}$.

Fig. $4 a$ shows the nontwisted 1 -form $d x+2 d y$ with inner orientation given by an arrow. Fig. $4 b$ shows the

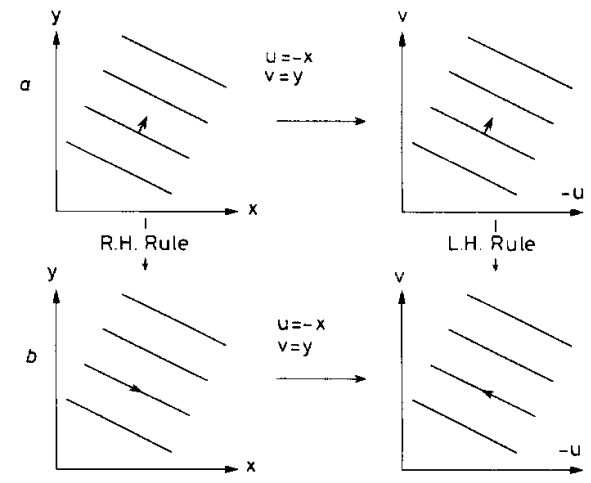

Fig. 4 (a) The behaviour of the nontwisted 1-form $d x+2 d y$ in the plane under a coordinate transform. (b) The behaviour of the twisted 1 -form $d x+2 d y$ under the same transform.

The orientations of the untransformed forms are related by the right-hand rule. Since the coordinate transform changes the handedness of the coordinate system, the orientations of the transformed forms are related by the left-hand rule, and so
the orientation of the twisted form reverses

twisted form $d x+2 d y$ in $R^{2}$. Its outer orientation with respect to $\Omega=d x d y$ is provided by the 1 -form $\frac{1}{5}(2 d x$ $-d y$ ), which has orientation in the direction of the arrow in Fig. $4 b$. Under coordinate reflection, the orientation of a twisted form reverses with respect to the orientation of the nontwisted form with the same components, as shown in the figure. 
Under pullback or restriction to a subspace*, the outer orientation $\left\{\left(\alpha_{s}, \Omega_{s}\right)\right\}$ of the twisted form $(\alpha, \Omega)$ restricted to a subspace with outer orientation $n$,

$$
\left\{\left(\alpha_{s}, \Omega_{s}\right)\right\} \wedge n=\{(\alpha, \Omega)\}
$$

where $\alpha_{s}$ is the restriction of the form $\alpha$ to a subspace and $\Omega_{s}$ is a volume element in the subspace. The 1-form $n=$ $d f / \sqrt{ }(d f\lrcorner d f)$ used in the preceding sections serves as the outer orientation for the boundary $f=0$, so that eqn. 39 and definition 38 lead to convention 10.

Twisted forms are the natural mathematical object to represent sources. Consider, for example, a surface current in the $+x$ direction along the $x-y$ plane. The usual vector $J_{s}$ for this current is $J_{0} \hat{x}$. The nontwisted 1 -form $J_{0} d x$ dual to $J_{0} \hat{x}$ has inner orientation in the $+x$ direction, but does not integrate over a path to yield the correct current through the path - the current through the $y$-axis from $y=0$ to $y=1$, for example, is $J_{0}$, but the integral of $J_{0} d x$ over that path is zero. The nontwisted 1 -form $J_{0} d y$ integrates properly to yield current through a path, but has inner orientation in the $+y$ direction. The twisted 1-form $\left(J_{0} d y, d x d y\right)$ by eqn. 38 has outer orientation $d x$, which is the direction of current flow, and

* This definition for the orientation of the restriction of a form to boundary, or pullback to the boundary, corresponds with the expected orientations of EM boundary conditions. For pullback to commute with exterior differentiation, however, the convention $n \wedge\left\{\left(\alpha_{s}, \Omega_{s}\right)\right\}=$ $\{(\alpha, \Omega)\}$ found in Burke [6] must be used intead of eqn. 39 (Burke, W.L. Private communication, Feb. 1995). This convention could be employed here if the surface normal 1-form were oriented from $D_{1}$ to $D_{2}$ as before but from $H_{2}$ to $H_{1}$. In fact, applying the continuity equation for surface charge and the comm Burke's boundary conditions using pullback, one can sh pullback functions for $H$ must differ from those for $D$ by a sign. We prefer to leave the boundary conditions simple at the expense of some required caution when using our definition of pullback more generally. integrates to yield the correct current through an arbitrary path as well.

Twisted 2-forms are similarly useful for representing surface charge. Each cell of a non-twisted 2-form in twospace has a screw sense as inner orientation, whereas each cell of a twisted 2-form has a sign as its outer orientation (Fig. 5).

We represent surface current and surface charge density with twisted forms because of the graphical convenience of having orientation of forms correspond to

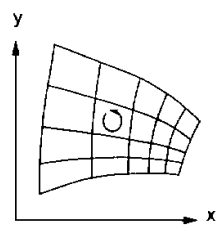

a

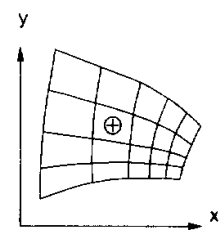

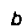

Fig. 5 (a) The inner orientation of a nontwisted 2-form $\beta$ in two-space, specified by a screw sense for each box. (b) The outer orientation of a twisted 2-form $(\beta, \Omega)$ in two-space is a sign for each box

actual direction of flow of current or sign of the charge. To be precise, $H$ and $D$ must be twisted forms also. For clarity's sake, we ignore this in the body of the paper, since as mentioned above, one can employ inner and outer orientations interchangeably if only right-handed coordinates are used. It is interesting to note that when properly formulated using both twisted and nontwisted forms as in [5], the 3+1 representation of electromagnetic field theory becomes explicitly parity invariant no right-hand rule is required. 